\title{
The role of indocyanine green fluorescence in endoscopic endonasal skull base surgery and its imaging correlations
}

\author{
Mostafa Shahein, MD,,3 Daniel M. Prevedello, MD,,2 Thomas L. Beaumont, MD, PhD, ${ }^{1}$ \\ Khalid Ismail, MD, ${ }^{3}$ Radwan Nouby, MD, ${ }^{3}$ Marilly Palettas, MPH, ${ }^{4}$ Luciano M. Prevedello, MD, MPH, ${ }^{5}$ \\ Bradley A. Otto, MD, ${ }^{1,2}$ and Ricardo L. Carrau, MD ${ }^{1,2}$ \\ Departments of ${ }^{1}$ Neurological Surgery, ${ }^{2}$ Otolaryngology-Head and Neck Surgery, ${ }^{4}$ Biomedical Informatics-Center for \\ Biostatistics, and ${ }^{5}$ Radiology, The Ohio State University Wexner Medical Center, Columbus, Ohio; and ${ }^{3}$ Department of \\ Neurosurgery, Aswan University Hospitals, Aswan University, Aswan, Egypt
}

\begin{abstract}
OBJECTIVE The use of endoscope-integrated indocyanine green (E-ICG) has recently been introduced in skull base surgery. The quantitative correlation between E-ICG and T1-weighted gadolinium-enhanced (T1WGd) images for skull base tumors has not been previously assessed, to the authors' knowledge. In this study, the authors investigated the indications for use and the limitations of E-ICG and sought to correlate the endoscopic fluorescence pattern with MRI contrast enhancement.
\end{abstract}

METHODS Following IRB approval, 20 patients undergoing endoscopic endonasal skull base surgery between June 2017 and August 2018 were enrolled in the study. Tumor fluorescence was measured using a blue color value and blood fluorescence as a control. Signal intensities (SIs) of tumor T1WGd images were measured and the internal carotid artery (ICA) SI was used as a control. For pituitary adenoma, the pituitary gland fluorescence was also measured. The relationships between ICG fluorescence and MRI enhancement measurements were analyzed.

RESULTS Data showed that in pituitary adenoma there was a strong correlation between the ratios of gland/blood fluorescence to gland $/ / C A S I(n=8 ; r=0.92 ; p=0.001)$ and tumor/blood fluorescence to tumor/lCA SI $(n=9 ; r=0.82 ; p=$ $0.006)$. In other pathologies there was a strong correlation between the ratios of tumor/blood fluorescence and tumorl ICA SI $(n=9 ; r=0.74 ; p=0.022)$. The ICG fluorescence allowed perfusion assessment of the pituitary gland as well as of the nasoseptal flaps. Visualization of the surrounding vasculature was also feasible.

CONCLUSIONS Defining the indications and understanding the limitations are critical for the effective use of E-ICG. Tumor fluorescence seems to correlate with preoperative MRI contrast enhancement.

https://thejns.org/doi/abs/10.3171/2020.6.JNS192775

KEYWORDS neuroendoscopy; skull base surgery; pituitary; indocyanine green; ICG

I NDOCYANINE green (ICG) has been used for decades in vascular neurosurgery since its introduction by Raabe et al. ${ }^{1}$ Several studies have described the benefits of using microscope-integrated ICG in various spinal and cranial tumors. ${ }^{2-6}$ Recent studies have suggested the administration of high doses of ICG the day before surgery to take advantage of the fluorescence induced in meningiomas, gliomas, and brain metastases. ${ }^{7-9}$

Endoscope-integrated ICG (E-ICG) has been used in visualizing vascular structure $\mathrm{s}^{10-13}$ and performing biopsy of intraventricular tumors..$^{14}$ In skull base surgery, E-ICG was first introduced to assess its feasibility in pituitary tumors. ${ }^{15}$ Adenomas were found to be hypofluorescent in comparison with the pituitary gland. ${ }^{15,16}$ Subsequent stud- ies measured the fluorescence of pituitary adenomas based on a blue color value, ${ }^{17,18}$ with results suggesting that better visualization is associated with higher fluorescence ratios of gland to tumor. ${ }^{18}$ In addition, E-ICG has been suggested as a tool to enhance visualization of vasculature, assessment of blood supply to nasoseptal flaps, and tumor identification. ${ }^{17,19-22}$

Some investigators have concluded that there is a correlation between tumor fluorescence and radiological enhancement on T1-weighted gadolinium-enhanced (T1WGd) MR images. ${ }^{2,9}$ To our knowledge, however, there have been no described criteria for the selection of cases in which E-ICG may be useful, and there has been no quantitative correlation of fluorescence to preoperative imaging.

ABBREVIATIONS E-ICG = endoscope-integrated ICG; ICA = internal carotid artery; ICG = indocyanine green; SI = signal intensity; T1WGd = T1-weighted gadoliniumenhanced.

SUBMITTED October 12, 2019. ACCEPTED June 18, 2020.

INCLUDE WHEN CITING Published online November 13, 2020; DOI: 10.3171/2020.6.JNS192775. 


\section{Methods}

\section{Design and Demographic Data}

A prospective cohort study of intraoperative E-ICG was conducted in patients undergoing endoscopic skull base surgery at our center between June 2017 and August 2018. The study was approved by the Institutional Review Board in accordance with the Health Insurance Portability and Accountability Act (HIPAA). In total, 20 patients (age range 20-70 years, median 47.5 years; 7 males and 13 females) were included after providing informed consent. We divided the enrolled patients into two subgroups based on pathology: patients with pituitary adenoma $(n=10)$ and those with other skull base pathologies $(\mathrm{n}=10)$. A total of 20 vials of ICG (25 mg each) were allowed for the study, 10 for each subgroup. In both subgroups, patients were enrolled consecutively. As the design of the study was to include 10 pituitary adenomas and 10 other tumors, the pituitary adenoma subgroup was finalized earlier during the period of enrollment as this condition occurred more frequently. Patient exclusion criteria included pregnancy and allergy to penicillin, sulfa, iodide, or any other dye. Tumor pathologies included the following: pituitary adenoma, including 1 carcinoma $(n=10)$; chordoma $(n=4)$; tuberculum sellae meningioma $(\mathrm{n}=3)$; chondrosarcoma $(\mathrm{n}=1)$; esthesioneuroblastoma $(\mathrm{n}=1)$; and Rathke's cleft cyst $(\mathrm{n}=1)$.

\section{Equipment and Technique}

A standard endoscopic approach was achieved depending on the type and site of pathology, varying between transsellar, transtubercular-transplanum, and transclival approaches. One vial of ICG (25 mg) was allowed for each patient. Each vial was diluted in $10 \mathrm{ml}$ of saline to reach a final concentration of $2.5 \mathrm{mg} / \mathrm{ml}$ administered in either a single bolus dose or 2 divided doses of $12.5 \mathrm{mg}$ each. ICG was administered once the surgical corridor and the exposure were completed and at the end of surgery if a second dose was needed. Standard $0^{\circ}$ and $45^{\circ}, 4$-mm-diameter, 18-cm endoscopes (Karl Storz Endoscopy America, Inc.) were used for surgery. For visualization of ICG fluorescence, a separate endoscope consisting of a Cold Light Fountain D-LIGHT P and an IMAGE1S H3-Z FI ThreeChip FULL HD camera attached to a 5.8-mm-diameter, 19-cm-length endoscope (all Karl Storz Endoscopy America, Inc.) was used. Shifting between the ICG and the normal mode was effected using a foot pedal.

\section{Video and Imaging Analysis}

A sample of how the measurements were achieved is presented in Fig. 1.

Fluorescent scenes were interpreted during surgery; in addition, all surgical videos were archived in full length and analyzed postoperatively. E-ICG was used at different stages during surgery depending on the aim of its utilization: 1) At the beginning of the tumor dissection, a continuous ICG mode was used to identify the parasellar or paraclival carotid artery, intercavernous sinus, basilar plexus, condition of nasoseptal flap, abnormal dural vasculature, and tumor or pituitary gland fluorescence; and in the middle of surgery, it was used to assess the fluores- cence of the tumors, pituitary gland, and stalk. 2) At the end of surgery, a second dose was used for the assessment of tumor residual or pituitary gland fluorescence and of the integrity of vascular structures in the tumor bed.

The latency of fluorescence signal onset was recorded by timing the start of the internal carotid artery (ICA) fluorescence, after ICG injection, which is the first structure to be fluorescent. A blue color value ranging from 0 to 255 , using Image ${ }^{23}$ software, was used to measure the fluorescence of the gland, tumor, and blood in pituitary adenoma cases and of the tumor and blood in other pathologies. Single or two divided doses were used in all cases. All the fluorescence measurements were taken only after the first ICG dose to avoid any possible additive effect.

Snapshots were generated from ICG surgical videos divided into 1-second intervals. The snapshots selected for blood fluorescence measurement were those obtained when maximum blue color value was reached in the blood. For pituitary adenoma cases, the snapshots selected for the gland and tumor fluorescence measurements were taken when the maximum blue color value was reached in the pituitary gland. For other tumor cases, the snapshots selected for the tumor fluorescence measurement were when the maximum blue color value was reached in the tumor. The distance of the endoscope from areas measured ranged from $1 \mathrm{~cm}$ to $2 \mathrm{~cm}$. The mean of a color range in a 40-pixel-diameter circular area (300 DPI) that was selected for each structure mentioned was used for the final statistical analysis. The circular area was selected from parts that were not contaminated with blood to avoid overlapping fluorescence.

On T1WGd images, the signal intensities (SIs) of the gland, tumor, and ICA in pituitary adenoma cases and of the tumor and ICA in other pathologies were measured using a Lexmark NilRead image viewer. Circular areas of $1-\mathrm{mm}$ and $2.5-\mathrm{mm}$ diameter for the ICA and tumor SI, respectively, were used for the measurement. The mean of the SI range in each circular area was used for the final statistical analysis.

\section{Statistical Methods}

Patient demographic characteristics were summarized using descriptive statistics. ICG fluorescence was expressed as the ratios of pituitary gland/blood and tumor/ blood blue color values in the pituitary adenoma subgroup and the ratio of tumor/blood blue color value in the other pathologies subgroup. MRI enhancement was expressed as the ratios of pituitary gland/ICA and tumor/ICA SI in the pituitary subgroup and the ratio of tumor/ICA SI in the other pathologies subgroup. Pearson's correlation coefficients were calculated to determine correlations between ICG fluorescence and MRI enhancement measures, respective to each subgroup. Analyses were performed in SAS 9.4 (SAS Institute) and in R version 3.5.2 (R Foundation for Statistical Computing).

\section{Results}

No adverse effects were noted related to the drug in a follow-up period of 6 months. The findings were categorized as reported in the following sections. 



FIG. 1. Measurement of the MRI SI and ICG fluorescence. A: Screenshot of axial T1WGd showing the measurement of the SI of both the pituitary gland and the pituitary adenoma. B: Screenshot of the image representing when the maximum blue color value was reached in the pituitary gland, with attached screenshots from the measurements generated using ImageJ software for both the pituitary gland and the tumor. All similar measurements in addition to the control measurements were grouped and ratios were calculated and then statistically analyzed. $G=$ gland; $T=$ tumor. Figure is available in color online only.

\section{Intraoperative Tumor Fluorescence (based on pathology) Pituitary Adenoma Subgroup}

In 8 of the 10 cases, E-ICG was useful and identification of the pituitary gland and the adenoma was successful. In 2 cases, E-ICG was not helpful (Video 1).

VIDEO 1. Sample of pituitary adenoma cases in patients enrolled in the study. $C S=$ cavernous sinus; $G$ = gland; $T$ = tumor. Copyright Daniel M. Prevedello. Used with permission. Click here to view.

The pituitary gland was found to be more fluorescent than the adenoma (Fig. 2). Careful interpretation of the ICG scenes in these cases was essential due to the other surrounding fluorescent structures.

We categorized our findings based on certain criteria that required careful consideration. Clear distinction between the gland and the tumor preoperatively on T1WGd images predicted helpful ICG scenes. However, in recurrent pituitary adenoma and pituitary carcinoma cases, E-ICG was not helpful as clear distinctions could not be found preoperatively on radiological imaging. The presence of an intact pseudocapsule, which represents a compressed pituitary gland and helps in extracapsular resection, ${ }^{24}$ and the absence of marked intratumoral bleeding made E-ICG helpful. In relatively large macroadenomas, the pseudocapsule was disrupted and intratumoral bleeding was more prominent. As the blood was fluorescent during the early phase of ICG injection, the fluorescent 

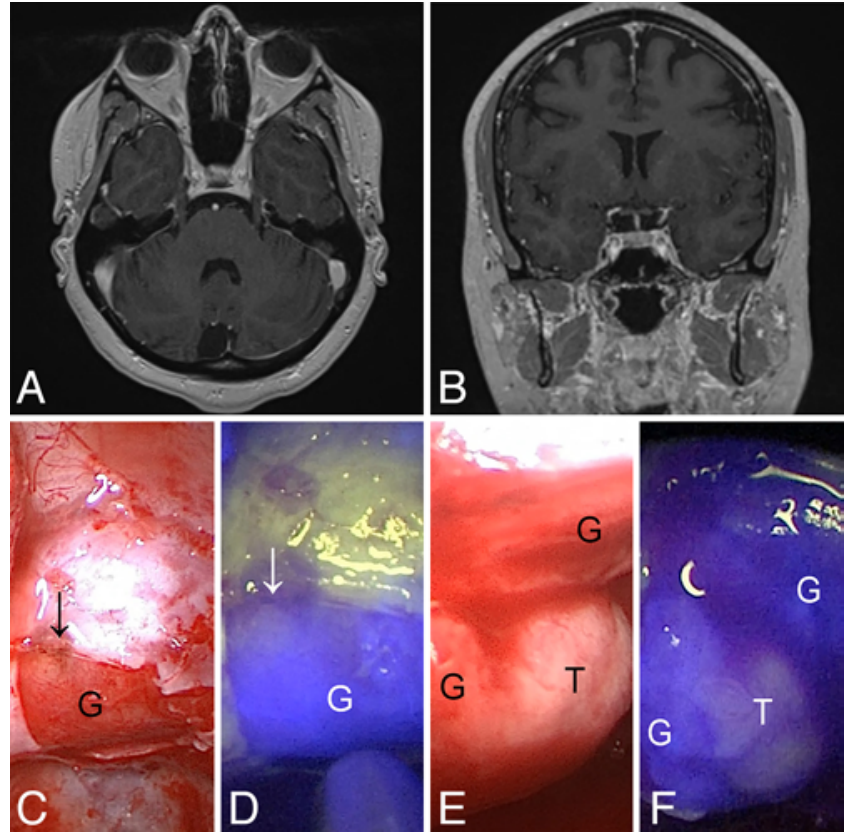

FIG. 2. Fluorescence of the tumors (pituitary adenoma)-T1WGd MR images. Axial (A) and coronal (B) cuts show pituitary microadenoma where localization might be difficult. The gland covering the whole sellar surface (C) and the fluorescent gland covering the tumor and initial incision based on the preoperative radiological images at the suction tip (D) are shown. Effects of minimal dural cautery reflected on the fluorescence are indicated $(\downarrow)$. The gland and tumor under an ordinary endoscope $(E)$ are well differentiated based on the pattern of the fluorescence obtained using E-ICG $(F) . G=$ gland; $T=$ tumor. Figure is available in color online only.

pituitary gland and nonfluorescent adenoma could not be easily distinguished. In these cases, the fluorescent pituitary gland was only visualized after clearance of ICG from the blood. Nonfluorescent residual tumor was encountered against surrounding fluorescent structures, such as the pituitary gland or the cavernous sinus. Finally, presentation at the sellar surface helped in early identification of the tumor/gland interface. The presence of a thinned normal pituitary gland covering the area exposed necessitated an initial incision based on the predicted location. E-ICG was helpful afterward and not initially during surgery. An integrated summary of our subjective remarks and proposed flowchart is shown in Fig. 3.

\section{Other Pathologies Subgroup}

Different findings were present and depended mainly on the preoperative imaging enhancement (Video 2).

VIDEO 2. Samples of tumors other than pituitary adenomas (chordoma and tuberculum sellae meningioma) in patients enrolled in the study. Copyright Daniel M. Prevedello. Used with permission. Click here to view.

Four cases of chordomas and 1 chondrosarcoma with different enhancement patterns were studied (Fig. 4). In the 4 chordoma cases, 2 demonstrated contrast enhancement on T1WGd images and were fluorescent using EICG. The tumor-specific fluorescence helped delineate the tumor and suspected small foci of tumor seedings around the tumor. In the other 2 cases, where there was weak or no enhancement of the tumor, weak or no fluorescence, respectively, was noted. The 1 chondrosarcoma case showed mixed enhancement radiologically, with the central part being neither enhancing nor fluorescent; however, the bilateral posterior clinoidal parts were radiologically enhancing and fluorescent with E-ICG.

All meningiomas in the study showed enhancement on T1WGd images. This finding correlated well with the tumor fluorescence seen on E-ICG. The site of the dural attachment of the meningiomas could be localized based on the abnormal dural vasculature viewable with E-ICG. After tumor resection, enhancing dural residuals could be detected for removal or cauterization.

\section{ICG Fluorescence/MRI Enhancement Correlation}

In the pituitary adenoma subgroup (Table 1), in each case the ratio of the blue color value (representing the ICG fluorescence) of the pituitary gland compared with that of the blood (mean \pm SD $0.722 \pm 0.13$ ) and of the tumor compared with the blood $(0.538 \pm 0.16)$ were compared, respectively, with the ratios of pituitary gland/ICA $(0.675 \pm$ $0.11)$ and tumor/ICA $(0.494 \pm 0.087)$ SIs (representing the MRI enhancement) in T1WGd images. The degree of the differential ICG fluorescence between the pituitary gland and the tumor was correlated to the degree of differential SI between the gland and tumor in the T1WGd images. The mean ratios of gland/tumor ICG fluorescence (1.476 $\pm 0.249)$ and gland/tumor MRI SI $(1.405 \pm 0.100)$ were calculated. In the other pathologies subgroup (Table 2), the tumor/blood blue color value ratio was compared with the tumor/ICA SI ratio for each case.

Scatterplots were used to visually display the ICG fluorescence and MRI SI correlations (Fig. 5). In the pituitary adenoma subgroup, there was a strong correlation between the pituitary gland/blood and gland/ICA ratios $(n=8 ; r=$ $0.92 ; \mathrm{p}=0.001$ ) and tumor/blood and tumor/ICA ratios (n $=9 ; \mathrm{r}=0.82 ; \mathrm{p}=0.006)$. However, negligible associations were detected for the differences between the pituitary gland/blood and tumor/blood ratios in images with ICG in comparison with the differences between the pituitary gland/ICA and tumor/ICA ratios in MR images $(n=8 ; r$ $=0.09 ; p=0.83)$. In the subgroup of patients with other pathologies, there was a strong correlation between the tumor/blood and tumor/ICA ratios $(n=9 ; r=0.74 ; p=0.22)$.

In the pituitary adenoma subgroup, comparison of the gland/blood and tumor/blood ICG fluorescence ratios revealed a statistically significant difference $(\mathrm{t}=4.39, \mathrm{p}=$ 0.001). In addition, comparison of the gland/ICA MRI SI and tumor/ICA MRI SI ratios revealed a statistically significant difference $(\mathrm{t}=11.33$, $\mathrm{p}<0.001)$.

Two cases were excluded from the correlation measurements. The first case was in a patient with a recurrent pituitary adenoma, which was excluded because there was no clear distinction between the ICG fluorescence and MRI SI measurements for the gland and tumor. The second was the Rathke's cleft cyst case, in which thinwall cyst fluorescence could not be accurately measured. The chondrosarcoma case had two values for each of the ICG scene and MR image measurements representing the two different fluorescence and enhancement patterns, 


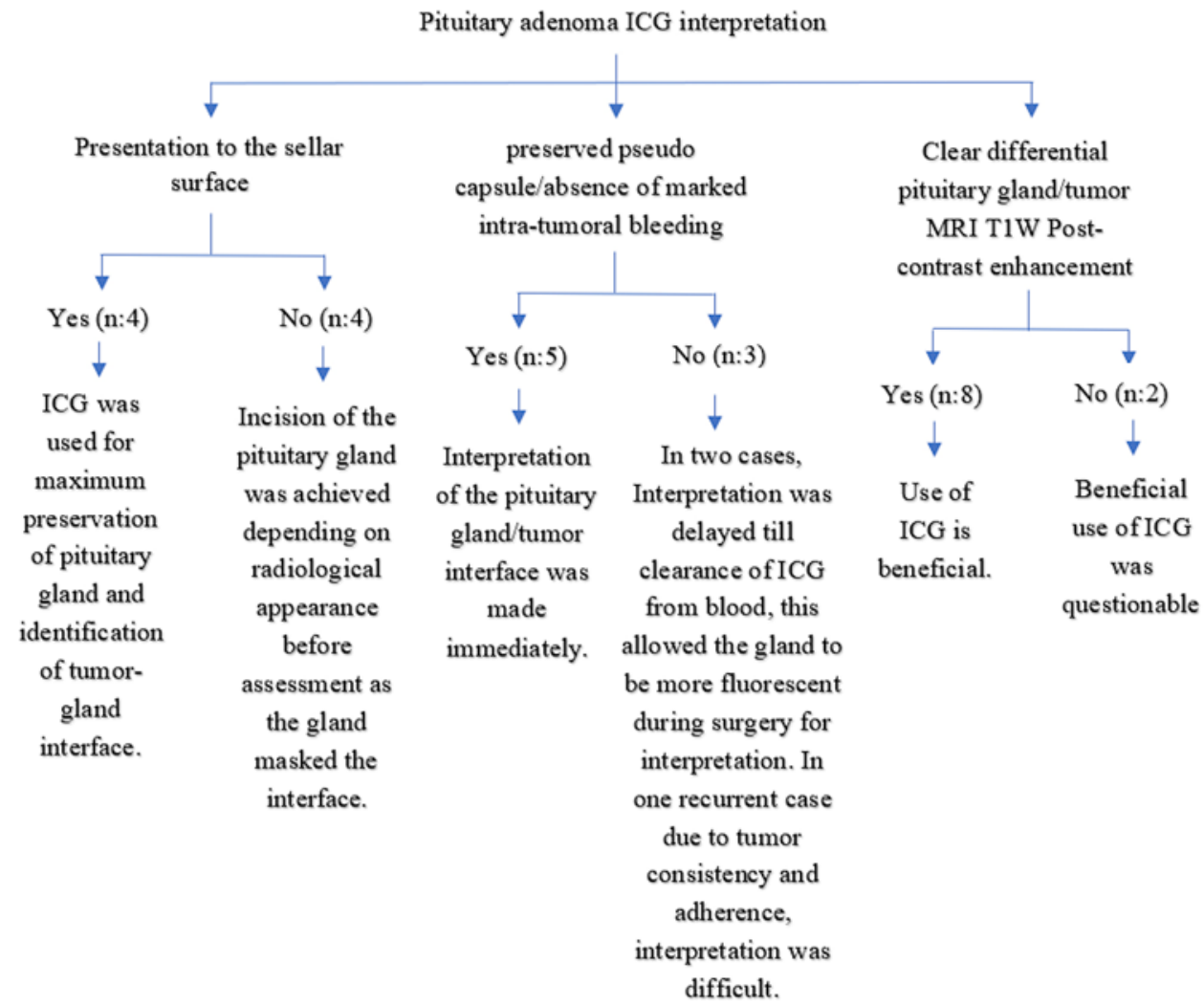

FIG. 3. A proposed flowchart with integrated data for the use of E-ICG in pituitary adenoma cases for which the perspective of the fluorescence of the tumor would be beneficial. Figure is available in color online only.

respectively; however, one value representing the center of the tumor was included for statistical analysis. In the pituitary carcinoma case, the aim of surgery was biopsy of the lesion as the patient had liver metastasis, so measurement of the pituitary gland fluorescence and SI was not achieved.

\section{Diffusion to Structures}

Visualization of the pituitary gland was helpful at different stages of surgery, especially in pathologies close to the gland. The gland was usually fluorescent for hours and fluorescence often lasted until the end of the surgery. In the Rathke's cleft cyst case that required pituitary gland splitting to reach the retrochiasmatic portion of the tumor, the fluorescence showed slower diffusion to the right side, possibly indicating decreased blood supply, in comparison with the left side. This matched the postoperative T1WGd images. The pituitary stalk and posterior lobe of the pituitary gland were also viewable in some cases.

The mucosal fluorescence helped in assessment of the condition of the nasoseptal flaps of 3 cases during the corridor stage. The first was a recurrent chordoma case, and the flap was elevated from its position, which was used in previous surgery. The flap demonstrated good flow based on the degree of the fluorescence signal with E-ICG and was reutilized. The second was a chordoma case in which there was unintentional partial violation of the pedicle of the nasoseptal flap, due to the nature of the extension of the tumor, and assessment of the flap condition using ICG demonstrated no fluorescent signal. The flap was reutilized and no CSF leak was encountered intra- or postoperatively. The third was an esthesioneuroblastoma case, in which there was a decreased flow in the harvested nasoseptal flap noted by the surgeons and confirmed with EICG in comparison with the surrounding normal mucosa. Multilayered reconstruction was needed, and no CSF leak was encountered postoperatively.

\section{Visualization of the Vasculature}

Before tumor dissection, different skull base vasculature could be viewed with ICG depending on the location of the pathology (Fig. 6). The ICA was the first structure to be fluorescent. Time from ICG injection to ICA fluorescence ranged from 3 to 48.85 seconds (average 18.125 seconds). The most rapid ICA fluorescence was in a chordoma case with a patent foramen ovale. E-ICG helped mainly in identification of the parasellar lateral limits of the approach in tuberculum sellae meningioma and pituitary adenoma cases. However, failed clear ICA detection was noted in 2 of 3 cases of acromegalic somatotrophs. Failed detection of the paraclival ICA was noted in 3 out of 5 clival lesions. Thickness of bone might be a reasonable explanation for this detection failure. In the pituitary carcinoma case, the tumor was completely surrounding the ICA and avidly fluorescent, which caused failure of ICA localization using E-ICG.

Other fluorescent vessels were the anterior and pos- 

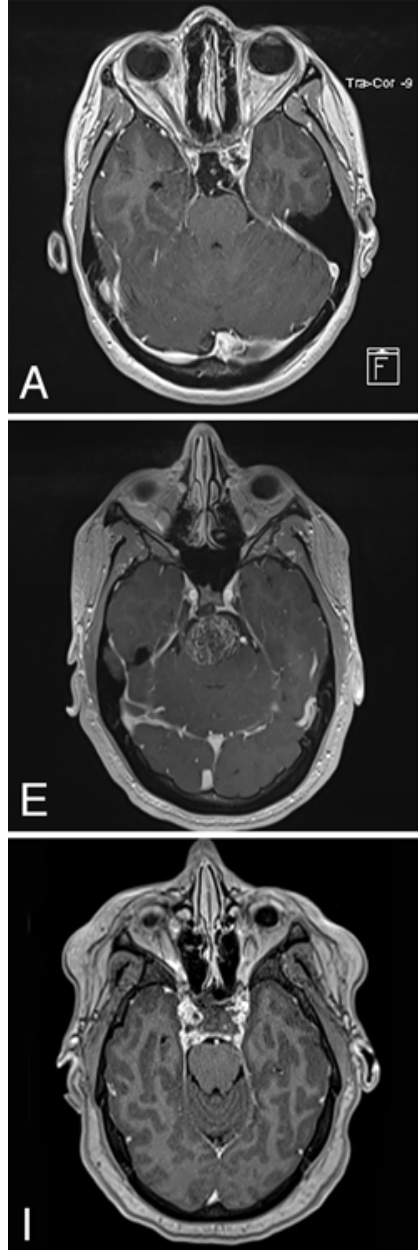
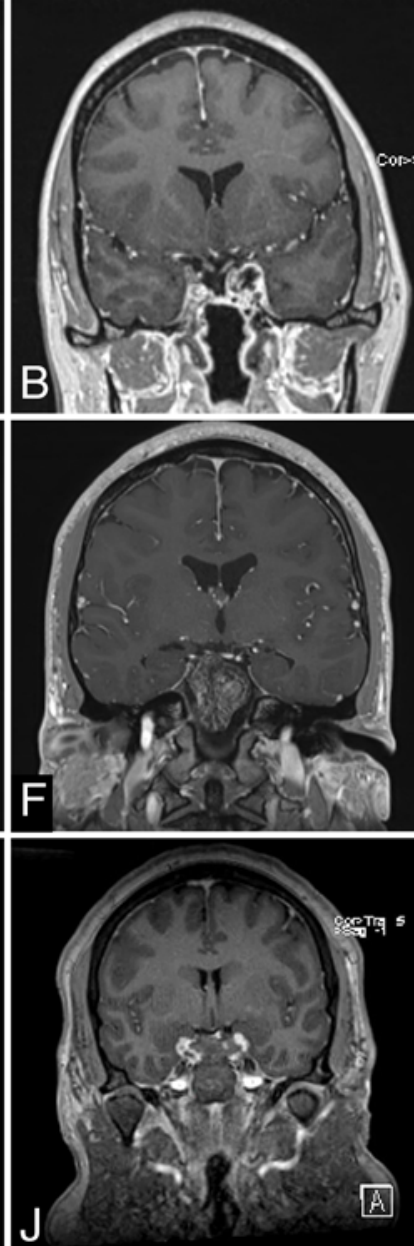
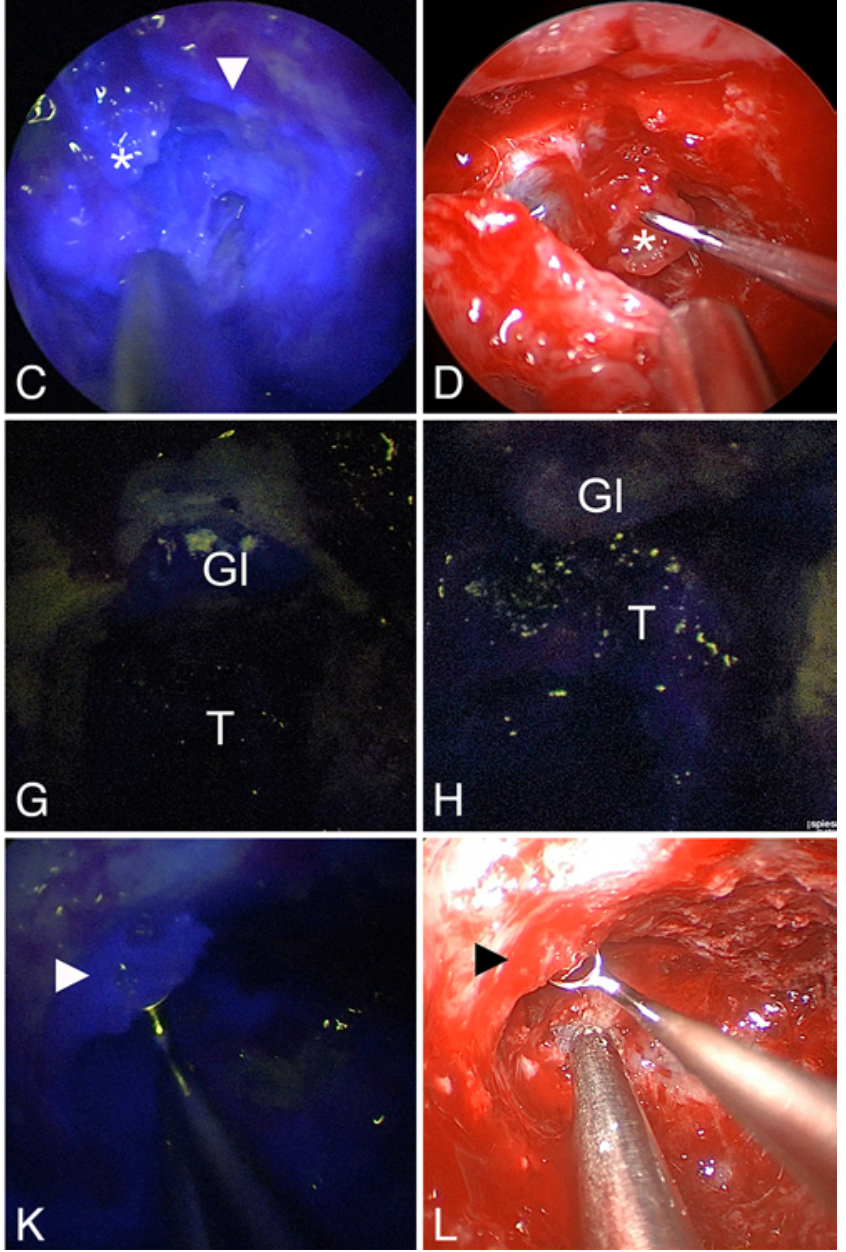

FIG. 4. Fluorescence of the tumors-chordoma and chondrosarcoma T1WGd MR images. Case 1. Axial (A) and coronal (B) cuts show left-sided recurrent chordoma, with the enhancing tumor on ICG mode $(\mathbf{C})$, with small seedings $(\boldsymbol{\nabla})$ that could be missed during resection in normal mode (D). Comparable parts on normal and ICG modes are indicated (*). Case 2. Axial (E) and coronal (F) cuts show a clival chordoma; the tumor after completion of the approach on ICG mode with the nonfluorescent tumor and the slightly fluorescent gland covered by the dura $(\mathbf{G})$. Later close inspection of very faint fluorescence of the tumor shown in panel $G$ $(\mathbf{H})$. Case 3. Axial $(\mathbf{I})$ and coronal $(\mathrm{J})$ cuts show the bilateral enhancing posterior clinoidal parts of the low-grade chondrosarcoma and the central hypoenhancing part, reflected in the ICG mode $(\mathbf{K})$ showing the fluorescent part in comparison with the normal mode $(\mathrm{L})(\triangleright)$. (By that time, the lack of a $45^{\circ} \mathrm{ICG}$ endoscope made the visualization of clinoidal parts difficult.) $\mathrm{GI}=$ gland; $\mathrm{T}=$ tumor. Figure is available in color online only.

terior ethmoidal vessels in the transcribriform approach. In this case, fluorescence helped in decreasing bleeding by accurate localization of such vessels. Abnormal dural blood vessels in meningiomas were also viewable with EICG, which helped in the localization and cauterization of feeding blood vessels before resection. McConnell's artery was noted to be hypertrophied and fluorescent in one case and was cauterized. Visualization of these vessels allowed the surgeons to achieve proper tumor devascularization. After tumor resection, vascular fluorescence helped in assessing the condition of the vessels in the tumor bed. In suprasellar lesions, the condition of the anterior cerebral arteries and the superior hypophyseal arteries could be detected. In clival lesions, E-ICG allowed assessment of the integrity of small and large brainstem perforators and also helped in assessing the flow in larger vessels, such as the basilar artery. Venous sinuses could also be viewable and assessed in some cases, including the cavernous sinus, intercavernous sinus, and basilar plexus.

\section{Discussion}

ICG with a special microscope has been used in the neurosurgical field for decades. ${ }^{1}$ Since the introduction of E-ICG, many articles have described the usefulness of this technology in various applications. ${ }^{10-21,25}$

The variations among studies of E-ICG in the skull base field, though relatively few, are notable. The dose of ICG varied between $5,{ }^{18} 6.25,{ }^{14} 12.5,{ }^{17}$ and 10 or $25^{15} \mathrm{mg} / \mathrm{dose}$. The timing for detection of the fluorescence in the pituitary gland ranged from 17 seconds ${ }^{17}$ to 10 minutes. ${ }^{14}$ Previous studies also showed variations in fluorescence timing of different structures. ${ }^{17}$ Quantification of the fluorescence was by either subjective ${ }^{15}$ or objective ${ }^{17,18}$ measurements. The tim- 
TABLE 1. Demographic and pathological data of the pituitary adenoma cases and postoperative measurements of the ratios of fluorescence and the intensity of both the gland and tumor in relation to the blood and ICA, respectively

\begin{tabular}{|c|c|c|c|c|c|c|c|c|c|}
\hline $\begin{array}{l}\text { Case } \\
\text { No.* }\end{array}$ & $\begin{array}{l}\text { Age (yrs)/ } \\
\text { Sex }\end{array}$ & $\begin{array}{c}\text { Pituitary Cell } \\
\text { Lineage }\end{array}$ & $\begin{array}{c}\text { Size (max } \\
\text { diameter, cm) }\end{array}$ & $\begin{array}{l}\text { A: Gland/Blood } \\
\text { Fluorescence }\end{array}$ & $\begin{array}{l}\text { B: Tumor/Blood } \\
\text { Fluorescence }\end{array}$ & $\begin{array}{l}\text { C: Gland/ICA } \\
\text { Intensity }\end{array}$ & $\begin{array}{l}\text { D: Tumor/lCA } \\
\text { Intensity }\end{array}$ & $\begin{array}{c}\text { Differential } \\
\text { Fluorescence } \\
\text { (A \& B)† }\end{array}$ & $\begin{array}{c}\text { Differential } \\
\text { Intensity } \\
(C \& D) \dagger\end{array}$ \\
\hline 1 & $38 / F$ & Corticotroph & 1.2 & 0.767 & 0.448 & 0.674 & 0.471 & 0.319 & 0.203 \\
\hline 2 & $70 / F$ & Gonadotroph & 2.1 & - & - & - & - & - & - \\
\hline 3 & $36 / \mathrm{M}$ & Somatotroph & 1.6 & 0.493 & 0.26 & 0.530 & 0.395 & 0.232 & 0.134 \\
\hline 4 & $70 / F$ & Null cell & 2.1 & 0.640 & 0.418 & 0.634 & 0.46 & 0.222 & 0.173 \\
\hline 5 & $54 / F$ & Corticotroph & 3.36 & - & 0.8 & - & 0.584 & - & - \\
\hline 6 & $25 / \mathrm{F}$ & Somatotroph & 2.9 & 0.569 & 0.469 & 0.534 & 0.36 & 0.1 & 0.174 \\
\hline 7 & $46 / F$ & Corticotroph & 0.7 & 0.778 & 0.638 & 0.772 & 0.522 & 0.14 & 0.249 \\
\hline 8 & $25 / F$ & Corticotroph & 0.4 & 0.861 & 0.658 & 0.828 & 0.592 & 0.203 & 0.236 \\
\hline 9 & $68 / \mathrm{M}$ & Somatotroph & 1.4 & 0.847 & 0.639 & 0.729 & 0.603 & 0.207 & 0.126 \\
\hline 10 & $57 / M$ & Gonadotroph & 1.6 & 0.814 & 0.507 & 0.699 & 0.485 & 0.307 & 0.24 \\
\hline Mean \pm SD & & & & $0.722 \pm 0.13$ & $0.538 \pm 0.16$ & $0.675 \pm 0.11$ & $0.494 \pm 0.087$ & & \\
\hline
\end{tabular}

Data based on measurements taken from surgical videos: A, ratios of the pituitary gland to blood ICG fluorescence; B, ratios of the pituitary adenoma to blood ICG fluorescence measurements. Data based on measurements taken from MRI: C, ratios of the pituitary gland to ICA signal intensity; D, ratios of the pituitary adenoma to ICA signal intensity.

${ }^{*}$ Case 2 was a recurrent pituitary adenoma that had no clear distinction between the pituitary gland and tumor. Case 5 proved to be a pituitary carcinoma with liver metastasis; the aim of surgery was biopsy and debulking, so pituitary gland visualization was not achieved.

† Statistically significant differences between $A$ and $B(t=4.39, p=0.001)$ and $C$ and $D(t=11.33, p<0.001)$

ing of ICA fluorescence was variable, which might be attributable to the differences among patient characteristics, including cardiac output and rate of clearance, as well as the rate of ICG injection. This justifies the fastest occurrence of
ICA fluorescence in a case with a patent foramen ovale in our series. For this reason, we found that setting a specific timing for visualization of each structure was not practical.

The gadolinium-based contrast agents are small par-

TABLE 2. Demographic and pathological data of the other tumor pathologies and the postoperative measurements of the ratios of fluorescence and the SI of the tumor in relation to the blood and ICA, respectively

\begin{tabular}{|c|c|c|c|c|}
\hline Tumor Type \& Case No. & Age (yrs)/Sex & Tumor Size, cm & A: Tumor/Blood Fluorescence & B: Tumor/ICA Intensity \\
\hline \multicolumn{5}{|l|}{ Chordoma } \\
\hline 1 & $34 / F$ & $1.2 \times 1 \times 1.2$ & 0.996 & 0.708 \\
\hline 2 & $20 / M$ & $3.4 \times 3.2 \times 4$ & 0.204 & 0.283 \\
\hline 3 & $70 / M$ & $5.7 \times 2.7 \times 7$ & 0.69 & 0.684 \\
\hline 4 & $38 / F$ & $1.1 \times 1 \times 1.3$ & 0.331 & 0.425 \\
\hline \multicolumn{5}{|l|}{ Meningioma } \\
\hline 1 & $49 / M$ & $0.9 \times 0.9 \times 0.6$ & 0.581 & 0.818 \\
\hline 2 & $67 / F$ & $0.5 \times 0.9 \times 0.8$ & 0.782 & 0.563 \\
\hline 3 & $57 / F$ & $2.1 \times 2 \times 2$ & 0.644 & 0.56 \\
\hline \multicolumn{5}{|l|}{ Chondrosarcoma } \\
\hline 1 & $57 / M$ & $3.1 \times 1.4 \times 4.7$ & $0.948,{ }^{*} 0.407 \dagger$ & $0.73,{ }^{*} 0.37 \dagger$ \\
\hline \multicolumn{5}{|l|}{ Esthesioneuroblastoma } \\
\hline 1 & $62 / M$ & $1.1 \times 0.6 \times 0.8$ & 0.486 & 0.564 \\
\hline \multicolumn{5}{|l|}{ Rathke's cleft cyst } \\
\hline 1 & $30 / F$ & $1.1 \times 1 \times 1.2, \ddagger 1 \times 0.9 \times 0.9 \S$ & - & - \\
\hline Mean \pm SD & & & $0.569 \pm 0.242$ & $0.553 \pm 0.171$ \\
\hline
\end{tabular}

A, ratio of tumor to blood ICG fluorescence measurements taken from surgical videos; B, ratio of tumor to ICA signal intensity measurements taken from MRI.

${ }^{*}$ Posterior clinoidal part of the tumor, not included in final statistical analysis.

$\dagger$ Central clival part of the tumor, included in final statistical analysis.

$\ddagger$ Sellar component of the cyst.

$\S$ Retrochiasmatic part of the cyst. 

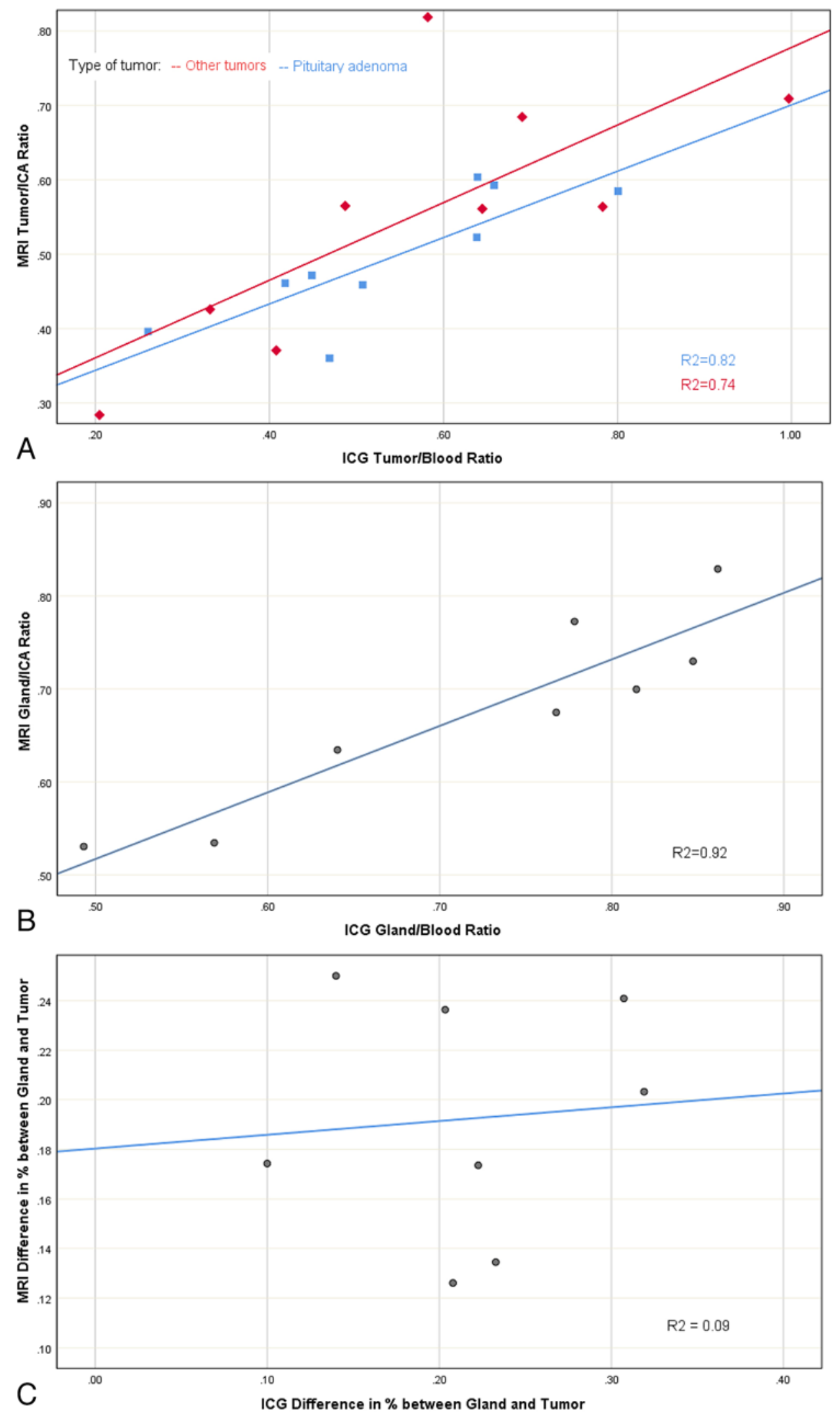

FIG. 5. Scatterplots showing the relationship between the ICG fluorescence and the MRI T1WGd SI measured postoperatively in the pituitary adenoma and other pathologies subgroups. A: The relationship between the MRI tumor/ICA SI ratio on the vertical axis and the tumor/blood ICG fluorescence ratio on the horizontal axis in both subgroups. FIG. 5. (continued) $\rightarrow$ 
FIG. 5. B: The relationship between the pituitary gland/ICA MRI SI ratio on the vertical axis and the pituitary gland/blood ICG fluorescence ratio on the horizontal axis in the pituitary adenoma subgroup. C: The relationship of the difference between the ratios of MRI gland/ICA and tumor/ICA SI on the vertical axis to the difference between the ratios of ICG gland/blood and tumor/blood fluorescence on the horizontal axis in the pituitary adenoma subgroup. Figure is available in color online only.

ticles that bind to the plasma protein and depend on defects in the blood-brain barrier to enhance the lesions on T1WGd images. ${ }^{26}$ These agents and ICG are hypothesized to share a similar mechanism of action. ${ }^{7}$ The correlation between the MRI enhancement and the ICG fluorescence pattern has been either rarely or unclearly mentioned in previous articles. ${ }^{2,9,17-19,27}$ To our knowledge, objective quantification of endoscopic ICG fluorescence was never correlated with the SI of MRI enhancement in the literature before the present study.
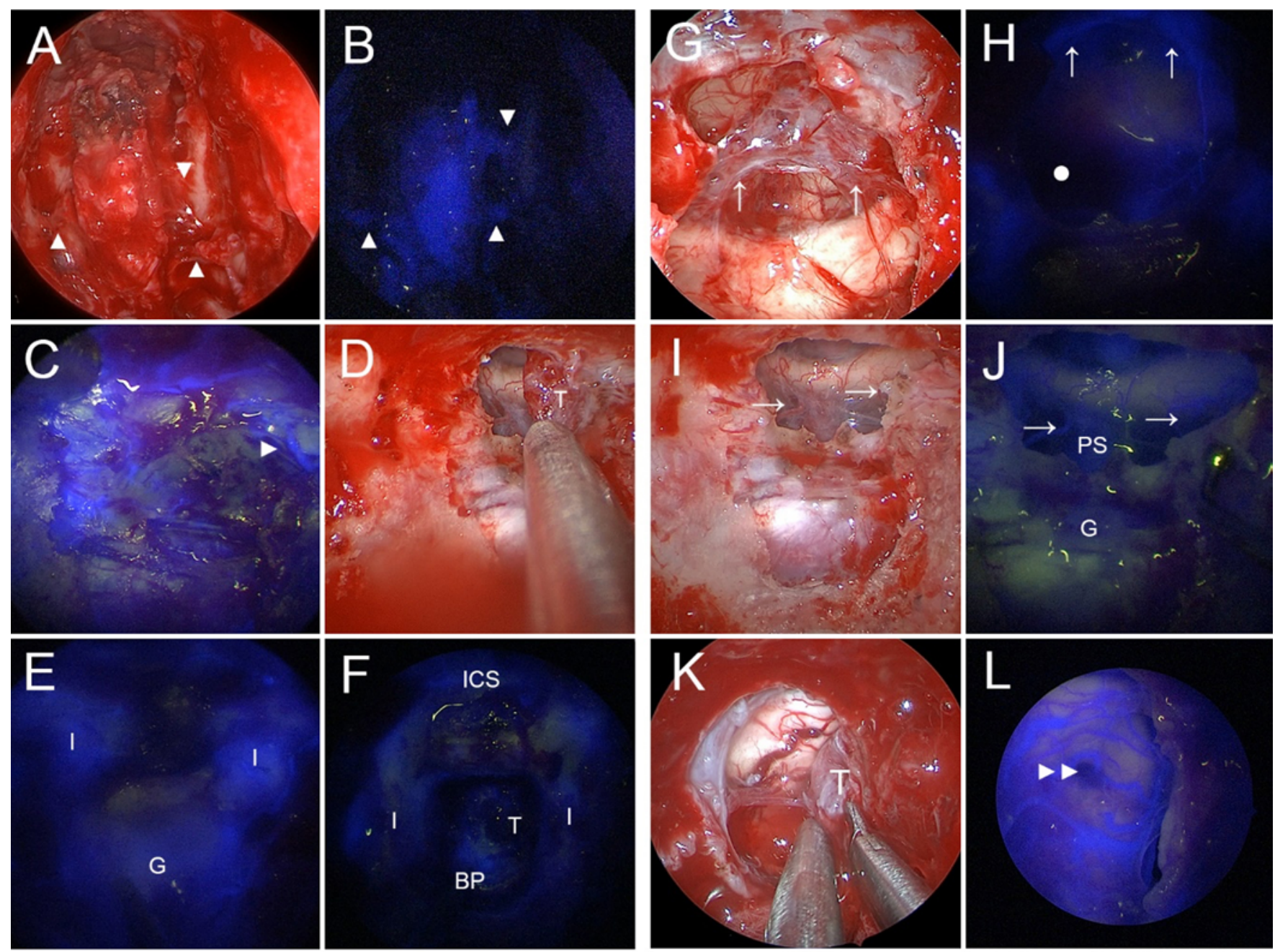

\section{Before tumor resection}

\section{After tumor resection}

FIG. 6. Visualization of the vasculature, before (left panels, A-F) and after (right pane/s, G-L) tumor resection. A: Transcribiform approach to a case of esthesioneuroblastoma in normal mode. B: ICG mode of the same case. The left anterior $(\boldsymbol{\nabla})$ and both posterior ethmoidal $(\boldsymbol{\Delta})$ arteries are marked in A and B. The left anterior ethmoidal artery was cauterized earlier. $\mathbf{C}$ : Transtubercular approach to a meningioma with a hypertrophied McConnell artery $(\boldsymbol{)})$ that needed cautery before dural opening and helped in decreasing the bleeding (D). E: Transtubercular approach after complete cautery of the dural attachment of a tuberculum sellae meningioma bordered by the fluorescent ICA on both sides. F: Transclival approach for a small chordoma with fluorescence of the surrounding vascular structures. G: A case of post-tuberculum sellae meningioma resection showing both anterior cerebral arteries $(\uparrow)$ using a $45^{\circ}$ endoscope. $\mathrm{H}$ : The view using a $0^{\circ}$ endoscope in ICG mode with fluorescent $(\uparrow)$ anterior cerebral arteries. Note that the blood clot $(\bullet)$ masked visualization of underlying structures. The stalk and superior hypophyseal arteries $(\rightarrow)$ are visualized on normal (I) and ICG (J) modes. K: A transclival approach for resection of a chordoma. L: Patency of the brainstem perforators and branches of the basilar artery in the tumor bed after resection. The small hypofluorescent portion $(\longrightarrow)$ represents a small part that was invaded by the tumor. BP = basilar plexus; $\mathrm{G}=$ gland; I = internal carotid artery; ICS = intercavernous sinus; $P S$ = pituitary stalk; $T$ = tuberculum sellae meningioma. Figure is available in color online only. 
We correlated the ICG fluorescence using the blue color value with the MRI T1WGd images using the SI. Each case was used as its own control, and ratios, instead of absolute values, were used for statistical analysis. Using this method helped in diminishing the possible variations related to each case, including pathological type and ICG characteristics. The degrees of the difference between the gland/tumor ICG fluorescence ratio and the gland/tumor MRI enhancement ratio are not correlated. This finding might be attributable to the different pathological subtypes and needs to be further investigated in subsequent studies.

In pituitary adenoma cases, the various patterns of enhancement seen in MR images are due to the different vasculature between the gland and the tumor. ${ }^{28}$ We always found a differential fluorescence, though variable, with the gland being more fluorescent. Based on our experience, we postulated that presentation to the sellar surface, presence of an intact pseudocapsule, and intratumoral bleeding were the main factors that should be considered in selection of the cases and the way of interpretation. In other types of tumors, E-ICG was helpful in fluorescent tumors that were enhancing radiologically. In nonfluorescent tumors, the lack of fluorescent background deemed the tumor detection unreliable. E-ICG might help in the future to achieve more precise resection, though the specificity and sensitivity of the exact tumor margins need further investigation with pathological confirmation.

In the perspective of visualization of the vascular structures, failure of ICA detection in some cases was due to limited penetration of the fluorescent ICG through tissues, which ranged from 2 to $10 \mathrm{~mm} .{ }^{7,15}$ Bone thickness, especially in patients with acromegaly or during assessment of the paraclival regions, and encasement by the tumor were the main obstacles. We believe that Doppler and navigation systems are still needed in addition to E-ICG for localization purposes.

In previous studies, authors concluded that E-ICG is better in visualization during aneurysm surgery ${ }^{29}$ or in pituitary surgery, ${ }^{30}$ attributing this to the closer distance of observation and better illumination. Such factors affected the degree of the blue color value, and this was ignored in many previous studies. However, using the ratios with similar distances for analysis made the effect of the distance on the blue color value not statistically significant. ${ }^{31}$

More technical advancement to help detect minor differences in the fluorescence will help in the differentiation of similarly fluorescent structures, especially in the cases of the pituitary adenomas. Different systems ${ }^{32}$ and technique ${ }^{27}$ can affect the ability to visualize ICG microscopically and consequently endoscopically. Real-time intraoperative correlation between the fluorescence and the enhancement may help in improving interpretation of the ICG scenes.

This study carries some limitations; there was no pathological confirmation of the fluorescent or nonfluorescent portions of the tumor to better identify the specificity, sensitivity, positive predictive value, and negative predictive value of the tumors' margins. Though these data are needed for idealization of the results in all the fluorescent materials, including ICG, 5-aminolevulinic acid, and sodium fluorescein, this method carries some controversy as it might lead to the risk of sampling normal tissue. ${ }^{33,34}$ This method also carries a higher risk of bias during sampling as the surgeon may be influenced by the concern of causing any harm to the patient. The current study stands as a preliminary step to expand and test the usefulness of ICG in specific pathologies separately. Subsequent separate specific studies including a larger number of patients for each pathology are needed.

\section{Conclusions}

E-ICG is a useful tool in skull base surgery. Careful consideration of the indications and identification of the limitations and imaging correlations help in selecting the patients who will benefit from the use of this method. The main purpose of this technique is to assess the vasculature around the tumor and its integrity and perfusion to normal structures or to stain the tumor itself.

\section{Acknowledgments}

This project has been supported in part by the Neuroscience Research Institute at The Ohio State University.

\section{References}

1. Raabe A, Beck J, Gerlach R, et al. Near-infrared indocyanine green video angiography: a new method for intraoperative assessment of vascular flow. Neurosurgery. 2003;52(1):132-139.

2. Acerbi F, Vetrano IG, Sattin T, et al. The role of indocyanine green videoangiography with FLOW 800 analysis for the surgical management of central nervous system tumors: an update. Neurosurg Focus. 2018;44(6):E6.

3. Takami T, Naito K, Yamagata T, et al. Benefits and limitations of indocyanine green fluorescent image-guided surgery for spinal intramedullary tumors. Oper Neurosurg (Hagerstown). 2017;13(6):746-754.

4. Asayama B, Sato K, Fukui T, et al. Skull bone tumor resection with intraoperative indocyanine green fluorescence imaging: a series of four surgical cases. Interdiscip Neurosurg. 2017;9:8-13.

5. Hojo M, Arakawa Y, Funaki T, et al. Usefulness of tumor blood flow imaging by intraoperative indocyanine green videoangiography in hemangioblastoma surgery. World Neurosurg. 2014;82(3-4):e495-e501.

6. Benedetto N, Aquila F, Vannozzi R. Use of near-infrared indocyanine videoangiography and Flow 800 in the resectioning of a spinal cord haemangioblastoma. Br J Neurosurg. 2013;27(6):847-849.

7. Lee JYK, Pierce JT, Thawani JP, et al. Near-infrared fluorescent image-guided surgery for intracranial meningioma. $J$ Neurosurg. 2018;128(2):380-390.

8. Lee JYK, Pierce JT, Zeh R, et al. Intraoperative near-infrared optical contrast can localize brain metastases. World Neurosurg. 2017;106:120-130.

9. Lee JYK, Thawani JP, Pierce J, et al. Intraoperative nearinfrared optical imaging can localize gadolinium-enhancing gliomas during surgery. Neurosurgery. 2016;79(6):856-871.

10. Wachter D, Behm T, von Eckardstein K, Rohde V. Indocyanine green angiography in endoscopic third ventriculostomy. Neurosurgery. 2013;73(1 Suppl Operative):ons67-ons73.

11. Bruneau M, Appelboom G, Rynkowski M, et al. Endoscopeintegrated ICG technology: first application during intracranial aneurysm surgery. Neurosurg Rev. 2013;36(1):77-85.

12. Mielke D, Malinova V, Rohde V. Comparison of intraoperative microscopic and endoscopic ICG angiography in aneurysm surgery. Neurosurgery. 2014;10(suppl 3):418-425. 
13. Yoshioka H, Kinouchi H. The roles of endoscope in aneurysmal surgery. Neurol Med Chir (Tokyo). 2015;55(6):469-478.

14. Tsuzuki S, Aihara Y, Eguchi S, et al. Application of indocyanine green (ICG) fluorescence for endoscopic biopsy of intraventricular tumors. Childs Nerv Syst. 2014;30(4):723-726.

15. Litvack ZN, Zada G, Laws ER Jr. Indocyanine green fluorescence endoscopy for visual differentiation of pituitary tumor from surrounding structures. J Neurosurg. 2012;116(5): 935-941.

16. Catapano G, Sgulò F, Laleva L, et al. Multimodal use of indocyanine green endoscopy in neurosurgery: a single-center experience and review of the literature. Neurosurg Rev. 2018; 41(4):985-998.

17. Hide T, Yano S, Shinojima N, Kuratsu J. Usefulness of the indocyanine green fluorescence endoscope in endonasal transsphenoidal surgery. J Neurosurg. 2015;122(5):1185-1192.

18. Verstegen MJT, Tummers QRJG, Schutte PJ, et al. Intraoperative identification of a normal pituitary gland and an adenoma using near-infrared fluorescence imaging and low-dose indocyanine green. Oper Neurosurg (Hagerstown). 2016;12(3):260-268.

19. Geltzeiler M, Nakassa ACI, Turner M, et al. Evaluation of intranasal flap perfusion by intraoperative indocyanine green fluorescence angiography. Oper Neurosurg (Hagerstown). 2018;15(6):672-676.

20. Inoue A, Ohnishi T, Kohno S, et al. Usefulness of an image fusion model using three-dimensional CT and MRI with indocyanine green fluorescence endoscopy as a multimodal assistant system in endoscopic transsphenoidal surgery. Int $J$ Endocrinol. 2015;2015:694273.

21. Kerr EE, Jamshidi A, Carrau RL, et al. Indocyanine green fluorescence to evaluate nasoseptal flap viability in endoscopic endonasal cranial base surgery. J Neurol Surg B Skull Base. 2017;78(5):408-412.

22. Simal Julián JA, Sanromán Álvarez P, Miranda Lloret P, Botella Asunción C. Endo ICG videoangiography: localizing the carotid artery in skull-base endonasal approaches. Acta Neurochir (Wien). 2016;158(7):1351-1353.

23. Schneider CA, Rasband WS, Eliceiri KW. NIH Image to ImageJ: 25 years of image analysis. Nat Methods. 2012;9(7): 671-675.

24. Oldfield EH, Vortmeyer AO. Development of a histological pseudocapsule and its use as a surgical capsule in the excision of pituitary tumors. J Neurosurg. 2006;104(1):7-19.

25. Yokoyama J, Ishibashi K, Shiramizu H, Ohba S. Impact of endoscopic indocyanine green fluorescence imaging on superselective intra-arterial chemotherapy for recurrent cancer of the skull base. Anticancer Res. 2016;36(7):3419-3424.

26. Provenzale JM, Mukundan S, Dewhirst M. The role of blood-brain barrier permeability in brain tumor imaging and therapeutics. AJR Am J Roentgenol. 2005;185(3):763-767.

27. Simal-Julián JA, Miranda-Lloret P, Evangelista-Zamora R, et al. Indocyanine green videoangiography methodological variations: review. Neurosurg Rev. 2015;38(1):49-57.

28. Di Ieva A, Weckman A, Di Michele J, et al. Microvascular morphometrics of the hypophysis and pituitary tumors: from bench to operating theatre. Microvasc Res. 2013;89:7-14.

29. Fischer G, Rediker J, Oertel J. Endoscope- versus microscope-integrated near-infrared indocyanine green videoangiography in aneurysm surgery. J Neurosurg. 2018;131(5): $1413-1422$

30. Sandow N, Klene W, Elbelt U, et al. Intraoperative indocyanine green videoangiography for identification of pituitary adenomas using a microscopic transsphenoidal approach. Pituitary. 2015;18(5):613-620.
31. Lee JYK, Cho SS, Zeh R, et al. Folate receptor overexpression can be visualized in real time during pituitary adenoma endoscopic transsphenoidal surgery with near-infrared imaging. J Neurosurg. 2018;129(2):390-403.

32. Cho SS, Zeh R, Pierce JT, et al. Comparison of near-infrared imaging camera systems for intracranial tumor detection. Mol Imaging Biol. 2018;20(2):213-220.

33. Stummer W, Pichlmeier U, Meinel T, et al. Fluorescenceguided surgery with 5-aminolevulinic acid for resection of malignant glioma: a randomised controlled multicentre phase III trial. Lancet Oncol. 2006;7(5):392-401.

34. Cho SS, Salinas R, Lee JYK. Indocyanine-green for fluorescence-guided surgery of brain tumors: evidence, techniques, and practical experience. Front Surg. 2019;6:11.

\section{Disclosures}

Storz provided the endoscopic equipment for ICG and the ICG vials. D. M. Prevedello reports being a consultant for Stryker, Medtronic, and Integra; being a patent holder in Mizuho, KLSMartin, and ACE-Medical; receiving royalties from KLS-Martin; and receiving honoraria from Mizuho and Storz.

\section{Author Contributions}

Conception and design: DM Prevedello, Shahein, Nouby, LM Prevedello, Carrau. Acquisition of data: DM Prevedello, Shahein, Beaumont, LM Prevedello, Otto. Analysis and interpretation of data: DM Prevedello, Shahein, Ismail. Drafting the article: Shahein. Critically revising the article: DM Prevedello, Ismail, Nouby, LM Prevedello, Otto, Carrau. Reviewed submitted version of manuscript: all authors. Approved the final version of the manuscript on behalf of all authors: DM Prevedello. Statistical analysis: Shahein, Palettas. Administrative/technical/material support: Shahein, Beaumont, LM Prevedello, Otto, Carrau. Study supervision: DM Prevedello, Carrau.

\section{Supplemental Information Videos}

Video 1. https://vimeo.com/436753965.

Video 2. https://vimeo.com/436753551.

\section{Previous Presentations}

Portions of the manuscript have been presented previously as a podium presentation at the 28th and 29th Annual Meetings of the North American Skull Base Society in San Diego, CA, February 16-18, 2018, and Orlando, FL, February 15-17, 2019. The abstracts have been published in J Neurol Surg B. 2018;79(S 01):S1-S188 and J Neurol Surg B. 2019;80(S 01):S1-S244.

\section{Correspondence}

Daniel M. Prevedello: The Ohio State University, Columbus, OH. daniel.prevedello@osumc.edu. 